\title{
Association between Metabolic Syndrome and Substance Dependence: A Cross-sectional Study in Kenya
}

SAMUEL MBUGUA ( $\square$ smungai.mbugua@gmail.com )

Mount Kenya University https://orcid.org/0000-0001-6611-9256

\section{Ezekiel Mecha}

University of Nairobi

\section{Peter Kirira}

Mount Kenya University

Margaret Njoroge

United States International University

\section{Boniface Malala}

Mount Kenya University

Jesse Gitaka

Mount Kenya University

Francis Makokha

Mount Kenya University

\section{Catherine Mwenda}

South Eastern Kenya University

\section{Research}

Keywords: Metabolic syndrome, substance dependence, anthropometric and biochemical measurements

Posted Date: January 6th, 2021

DOI: https://doi.org/10.21203/rs.3.rs-139422/v1

License: (1) (1) This work is licensed under a Creative Commons Attribution 4.0 International License. Read Full License 


\section{Abstract}

Background: Metabolic syndrome and its defining components remain an understudied area of human health research in Kenya and Africa. Understanding the relationship between substance dependence and the occurrence of metabolic syndrome is critical in prevention and clinical management of the related complications.

Methods: This was a cross-sectional study in 6 rehabilitative centers in 3 counties in Kenya with a conveniently selected sample size of 166 participants. A signed informed consent was obtained from each participant following which anthropometric and biochemical measurements were obtained. Descriptive statistics and chi-square test were used to describe the prevalence of metabolic syndrome and the relationship of the defining criteria with the substance of dependence.

Results: A quarter of the respondents were overweight and $6 \%$ obese. Fasting blood glucose was elevated in $62 \%$ of the sampled population with triglycerides having a significant variation with a mean of $216.95 \mathrm{mg} / \mathrm{dl}$ and a standard deviation of 151.107 . A prevalence rate of $4.8 \%$ was established based on the Harmonized Joint Scientific Statement on Metabolic syndrome for the African region. $87.34 \%$ of the population showed at least one elevated defining criteria with alcohol as the most prevalent substance of dependence. There was statistical difference of fasting blood glucose and triglycerides with alcohol use.

Conclusion: The findings indicate that alcohol use resulted to elevated levels of fasting blood glucose and triglycerides. There is need for emphasis on a multidisciplinary approach to substance dependence and metabolic syndrome management integrating physical activity interventions, dietary modifications and psychotherapeutic approaches.

\section{Background}

Metabolic syndrome is the co-existence of delineated clinical and biochemical risk factors that increases the propensity of having a cardiovascular disease or event by about three-fold [1]. It's a complex disorder defined by a cluster of inter-related factors that increase the proclivity of cardiovascular atherosclerotic diseases and type 2 diabetes. Metabolic syndrome remains a subject of considerable curiosity associated with the complexity of its pathophysiology [2].

The Joint Interim Statement of the International Diabetes Federation Task Force on Epidemiology and Prevention; National Heart, Lung, and Blood Institute; American Heart Association; World Heart Federation; International Atherosclerosis Society; and International Association for the Study of Obesity defined metabolic syndrome as elevated waist circumference and any of the following; elevated triglycerides, reduced High Density Lipoproteins (HDC), elevated blood pressure and elevated fasting blood glucose [3]. Primary preventive methods such as lifestyle changes are highly effective in improvement and preservation of cardiovascular health [4]. 
Substance use has a significant impact on the quality of life of families and children, and on nations and health systems [5]. The 2016 report on the United States National Survey of Drug Use and Health (NSDUH) estimated that 19 million adults above 18 years had a substance use disorder during the past year and 8.2 million had a substance use disorder and mental illness. In general, the numbers of adults initiating cocaine, cannabis and heroin increased in 2018 [6]. Substance use has shown increment in the risk of metabolic syndrome and diabetes in part due to increased cell damage and lowered cellular antioxidant potential [7]. Patients with metabolic syndrome have a higher occurrence of psychiatric comorbidity with psychotropics leading to development of metabolic syndrome [8]. Global estimates of the use of opioids is 28.6 million-38 million with a global prevalence of $0.7 \%[7,9]$. Increasing evidence indicates that opioids play a critical role in regulation of food intake and the reward basis based on ingestion [7].

Substance use prevention has a strong association to the constructs of social cognitive theory, the theoretical basis for explaining substance use [4]. Mattoo et al. [10] found a significant correlation of Body Mass Index (BMI) with presence of metabolic syndrome. The critical role of anthropometric measurements such as weight, waist circumference and BMI is emphasized by Mattoo et al. [11] and Grover et al. [12] who reported higher levels of BMI and obesity as significant predictors of metabolic syndrome. This is reinforced by findings from Gardner-Sood et al. [13] which emphasize the need for routine monitoring of waist circumference in psychosis and assertive management for cardiovascular risk in people with psychosis.

Understanding the causality and relationship of substance use and metabolic syndrome is critical towards development of definitive management and preventive measures among substance dependent persons. Metabolic syndrome and its defining elements in substance dependence remains an understudied research area in Kenya and Sub Saharan Africa despite the associated cardiovascular complications that manifest in these patients. The findings reported here are part of the project findings titled Gene-environment interactions in the genesis of substance dependence, psychosis and relapse in Kenya.

\section{Methods}

\section{Study sites and Design}

This was a cross-sectional study in 3 counties in Kenya; Mombasa, Muranga and Uasin Gishu. This was designed to sample a representative population of the country into the study due to the diversity in distribution of clients admitted in these rehabilitation centers. The study sites were six rehabilitation centers located in the three counties, two in each county. Community sensitization within counties hosting the study sites was done to encourage persons dependent on alcohol, cannabis and cocaine to visit the selected study rehabilitation centers. Samples were collected from consenting persons attending health facilities, admitted in rehabilitation centers and those sampled out from the community in the 
county who accepted to visit community sampling centers that were established in various parts of the country during the project.

Blood samples were collected from consenting individuals diagnosed as dependent to a substance or those who confess to be users and willingly visited the community sampling centers.

\section{Sampling}

A convenient sample of 166 substance patients was used in the study selected from 6 participating rehabilitation centers and the surrounding community sampling centers. Purposive sampling was used to select the 6 rehabilitation sites. Severe cases were referred to selected rehabilitation facilities for further management. Intervention mechanisms were also put in place to provide treatment and counselling collaboratively with selected rehabilitation centers as per the current standard of care.

\section{Anthropometric measurements}

Height, weight, waist circumference and blood pressure measurements were taken during a private physical examination by a qualified nurse. The height was measured using a Seca Rod 220 stadiometer (Seca, Hamburg, Germany), a TANITA (TANITA, Arlington Heights, Illinois) weighing scale to measure the weight and a Gulick tape measure to measure the waist circumference. All anthropometric measurements were taken without shoes and wearing light clothing. Waist circumference was measured at the iliac crest upon exhalation. Blood pressure was taken after a five minutes rest using an Omron automated monitor (Omron, Kyoto, Japan). The WHO guidance on body mass index classification was employed.

\section{Biochemical measurements}

SD Cholesterol/Lipid desktop analyzer was used to measure the biochemical measures for triglycerides, High Density Lipoproteins, and fasting glucose. Blood samples were acquired from a finger prick using a lancet and collected using a pipette. 12 hour fasting blood samples were assayed by standard methods for blood glucose, HDL-cholesterol and triglycerides in three minutes. A copy of the results was availed to each participant. If the results were outside normal parameters, the participant was referred for follow up and continued management with a health provider.

\section{Metabolic and its elements' defining criteria}

The defining criteria stipulated in the Harmonized Joint Scientific Statement (HJSS) [3] on metabolic syndrome was used. This requires central obesity or an elevated waist circumference and at least two other components. Precisely, the criteria is as follows: Waist circumference: $>94 \mathrm{~cm}$ (male) $>80 \mathrm{~cm}$ (female), Blood pressure- > 130/85 mmHg. (Hypertension), Impaired glucose handling-PG >200 mg/dL 
(11.1 mmol/L), raised fasting plasma glucose $>100 \mathrm{mg} / \mathrm{dL}(>5.6 \mathrm{mmol} / \mathrm{L})$, Body mass index $\geq 30 \mathrm{~kg} / \mathrm{m}^{2}$, Dyslipidemia: triglycerides (TG): $\geq 1.7 \mathrm{mmol} / \mathrm{L} / 150 \mathrm{mg} / \mathrm{dL}$ and high-density lipoprotein cholesterol $(\mathrm{HDL}-\mathrm{C}) \leq 1.0 \mathrm{mmol} / \mathrm{L} / 40 \mathrm{mg} / \mathrm{dL}$ (male), $\leq 1.3 \mathrm{mmol} / \mathrm{L} / 50 \mathrm{mg} / \mathrm{dL}$ (female).

\section{Results}

\section{Demographic characteristics}

The findings indicate that out of 166 rehabilitated patients participating in the study, majority of the respondents were male with $93.4 \%$ (155) and 6.6\% (11) were female. Most of the findings reported herein will be inclined towards the male gender. The mean age was 35.5 years $(S D=10.68)$ It was further established that $6.6 \%$ were aged $18-20$ years, $31.3 \%$ aged $21-30$ years, $31.3 \%$ aged $31-40$ years, $20.5 \%$ aged $41-50$ years and $6.6 \%$ above 50 years. $3.6 \%$ had missing information on the age. Regarding the level of academic achievement, $12.7 \%$ got to primary/elementary school level, $25.3 \%$ in high school, $1.2 \%$ in certificate level, and $22.3 \%$ in Diploma, $23.5 \%$ reached the degree level while $2.4 \%$ had masters. This indicates that majority of the substance abuse clients had attained at least high school completion.

\section{Anthropometric, clinical, and biochemical description of the participants}

The mean height and weight were 1.72 meters $(S D=0.078)$ and $69.5 \mathrm{~kg}(S D=12.63)$ respectively. The mean Body Mass Index was borderline normal at $23.46 \mathrm{~kg} / \mathrm{m}^{2}(\mathrm{SD}=4.05)$ with $24.7 \%$ reported as overweight and $6 \%$ as obese (Table 1). The mean waist circumference was $90.1 \mathrm{~cm}$ with $28.8 \%$ having central obesity. The study established a mildly elevated mean systolic blood pressure of 129.18 (SD = $16.240) \mathrm{mmHg}$. As for the diastolic blood pressure, they recorded a mean of 82.86 (SD =11.415). An elevated mean of $6.83 \mathrm{mmol} / \mathrm{L}(\mathrm{SD}=6.205)$ was recorded in fasting blood glucose with the standard deviation indicating a vast population spread and variability with $62 \%$ of the sampled population had elevated fasting blood glucose. This is an indication of the presence of prediabetes in majority of the population prone to substance abuse. Triglycerides revealed the most significant variability with a mean of $216.95 \mathrm{mg} / \mathrm{dl}$ and a standard deviation of 151.107 . The recorded mean of high-density lipoproteins was $54.20(\mathrm{SD}=39.862) \mathrm{mg} / \mathrm{dl}$ and a mean of $77.008(\mathrm{SD}=41.1888) \mathrm{mg} / \mathrm{dl}$ for low-density lipoprotein cholesterol. Non-high density lipoprotein cholesterol registered a mean of $115.23(S D=43.449) \mathrm{mg} / \mathrm{dl}$.

\section{Prevalence of Metabolic syndrome and each of the defining elements}

The prevalence rate of metabolic syndrome among substance abuse clients in rehabilitation in Kenya is $4.8 \%$ (8) out of a sampled population of 166 respondents. Most respondents with metabolic syndrome were between the ages of $31-40$ with $62.5 \%$ (5) with the males accounting for $75 \%$ (6) and females $25 \%$ 
(2). In relation to substance abuse and diagnosed metabolic syndrome, 75\% (6) used alcohol, $62.5 \%$ (5) used cannabis while $25 \%$ (2) used heroin.

Of the 166 valid responses from the patients undergoing rehabilitation, $87.34 \%$ had at least 1 component of the metabolic syndrome markers in all the 3 counties. The study established that $62.0 \%$ had impaired fasting glucose $42.2 \%$ had high systolic blood pressure, $37.3 \%$ had high diastolic blood pressure and $32.5 \%$ had elevated triglycerides. Hence, impaired fasting glucose was the most prevalent defining criteria for metabolic syndrome, followed by high blood pressure as indicated in Table 1.

Table 1

Prevalence of metabolic syndrome elements

\begin{tabular}{|llll|}
\hline Anthropometric/biochemical indices & \multicolumn{2}{l}{$\%$ of the population sampled } \\
\cline { 2 - 4 } & Normal & Elevated/Decreased & No recording \\
\hline Waist Circumference & 69.9 & 28.9 & 1.2 \\
\hline BMI & 60.2 & Overweight-24.7, & 1.2 \\
& & Obese-6 & \\
Systolic Blood Pressure & 56 & 42.2 & 1.8 \\
\hline Diastolic Blood Pressure & 60.8 & 37.3 & 1.8 \\
\hline Fasting Blood Glucose & 34.9 & 62 & 3 \\
\hline Triglycerides & 33.7 & 32.7 & $33.7^{\star}$ \\
\hline HDL-C & 53.6 & 12 (low) & $34.3^{*}$ \\
\hline $\begin{array}{l}* \text { The high percentage of respondents without readings as a result of refusal to have blood drawn for } \\
\text { the lipid profiling. }\end{array}$ & & \\
\hline
\end{tabular}

It was further established that $28.9 \%$ of the participants had a high waist circumference and $6.0 \%$ were obese. These results indicate that high waist circumference and obesity were the least prevalent defining criteria for cardiometabolic syndrome respectively.

Alcohol was the most prevalent substance of addiction at $86.2 \%$. The study sought to examine use of alcohol regarding the number of defining criteria using chi square test. The difference in fasting blood glucose levels and Triglycerides due to alcohol consumption was statistically significant $(P<0.05)$ with $67.41 \%$ and $54.74 \%$ of alcohol users showing elevated levels of these metabolic syndrome elements respectively. The conclusion drawn is that alcohol consumption mostly likely affected the levels of fasting blood glucose and triglycerides for the users. 
Table 2

Alcohol differences in regard to elevated metabolic syndrome elements

\begin{tabular}{|c|c|c|}
\hline MetS Criteria & $\%$ that reported alcohol abuse & $\begin{array}{l}X^{2} \\
\text { P Value }\end{array}$ \\
\hline \multirow[t]{3}{*}{ BMI } & \multirow[t]{3}{*}{$5.1 \%$} & $\chi^{2}=2.512^{a}$ \\
\hline & & $P=0.473$ \\
\hline & & $\mathrm{df}=3$ \\
\hline \multirow[t]{3}{*}{ Waist circumference } & \multirow[t]{3}{*}{$29.2 \%$} & $x^{2}=0.709^{a}$ \\
\hline & & $P=0.400$ \\
\hline & & $\mathrm{df}=1$ \\
\hline \multirow[t]{3}{*}{ Fasting blood glucose } & \multirow[t]{3}{*}{$67.41 \%$} & $\chi^{2}=4.880^{a}$ \\
\hline & & $P=0.027$ \\
\hline & & $d f=1$ \\
\hline \multirow[t]{3}{*}{ Triglycerides } & \multirow[t]{3}{*}{$54.74 \%$} & $x^{2}=9.177^{a}$ \\
\hline & & $P=0.002$ \\
\hline & & $d f=1$ \\
\hline \multirow[t]{3}{*}{ HDL-C } & \multirow[t]{3}{*}{$82.8 \%$} & $\chi^{2}=1.371^{a}$ \\
\hline & & $P=0.242$ \\
\hline & & $d f=1$ \\
\hline
\end{tabular}

Comparing this with two of the substances with higher prevalence rates, cannabis and cocaine, none of the metabolic syndrome elements were statistically significant as the p-value was more than 0.05 . Hence, there was no significant association between the use of cannabis and cocaine; and any of the metabolic syndrome elements. 
Table 3

Cannabis and cocaine differences in regard to elevated metabolic syndrome elements

\begin{tabular}{|c|c|c|c|c|}
\hline \multirow[t]{2}{*}{ MetS Criteria } & \multirow[t]{2}{*}{$\%$ abusing Cannabis } & \multirow[t]{2}{*}{$\%$ abusing cocaine } & \multirow{2}{*}{$\begin{array}{l}X^{2} \\
\text { Cannabis }\end{array}$} & \multirow{2}{*}{$\begin{array}{l}X^{2} \\
\text { Cocaine }\end{array}$} \\
\hline & & & & \\
\hline \multirow[t]{3}{*}{ BMI } & $7.23 \%$ & 0 & $x^{2}=3.300^{a}$ & $x^{2}=1.122^{a}$ \\
\hline & & & $P=0.348$ & $P=0.772$ \\
\hline & & & $d f=3$ & $d f=3$ \\
\hline \multirow[t]{3}{*}{ Waist circumference } & $24.10 \%$ & $40 \%$ & $\chi^{2}=1.099^{a}$ & $\chi^{2}=.343^{a}$ \\
\hline & & & $P=0.294$ & $P=0.558$ \\
\hline & & & $d f=1$ & $d f=1$ \\
\hline \multirow[t]{3}{*}{ Fasting blood glucose } & $60.98 \%$ & $60 \%$ & $\chi 2=146^{a}$ & $\chi^{2}=026^{a}$ \\
\hline & & & $P=0.703$ & $P=0.873$ \\
\hline & & & $d f=1$ & $\mathrm{df}=1$ \\
\hline \multirow[t]{3}{*}{ Triglycerides } & $50.0 \%$ & $66.67 \%$ & $\chi^{2}=0.877^{a}$ & $x^{2}=0.343^{a}$ \\
\hline & & & $P=0.349$ & $P=0.558$ \\
\hline & & & $d f=1$ & $d f=1$ \\
\hline \multirow[t]{3}{*}{ HDL-C } & $80 \%$ & $100 \%$ & $x^{2}=.014^{a}$ & $\chi^{2}=.763^{a}$ \\
\hline & & & $P=0.907$ & $P=0.382$ \\
\hline & & & $d f=1$ & $d f=1$ \\
\hline
\end{tabular}

\section{Discussion}

There exists a gap in cardiovascular and mental health research in Africa into the relationship between substance abuse and occurrence of metabolic syndrome and its elements. Substance abuse is a growing societal and health systems problem that requires urgent intervention. Part of the overall net effect in substance dependent individuals is a cascade of cardiovascular related risk factors denoting metabolic syndrome. Substance abuse patients have poorer diabetes and cardiovascular outcomes that include poor follow up, sub-optimal self-management skills and limited access to care [14, 15].

The prevalence rate of $4.8 \%$ observed in this project falls near the global range of $5 \%-31 \%$ in other studies of patients with alcohol abuse [11,16-19]. The prevalence of metabolic syndrome in psychiatric patients was significantly higher in Brazil at 29.4\% [20]. The most prevalent metabolic syndrome element was 
impairment of fasting glucose. This is different from observation by Mattoo et al. [11] and Yoon et al. [18] who identified elevated triglycerides as the most prevalent metabolic syndrome element.

A significant statistical difference is highlighted in the differences in triglyceride and fasting blood glucose levels resultant from alcohol consumption. This correlates with findings from Ojo et al., [21] who reported that the effect of substance abuse on fasting blood glucose was significant. Approximately a third of the sampled population had increased waist circumference with $12 \%$ having low HDL. These findings correlate with those of Shah et al. [22] who showed that history of ever use of any drug was associated with increased odds of elevated waist circumference and decreased odds of having low HDL.

Cannabis and cocaine users did not show any statistical difference in the elevated metabolic syndrome elements. Existing literature indicates that cannabis users have better metabolic profiles, $77 \%$ less likely to have metabolic syndrome according to Yankey et al. [2]. Cannabis use has also been associated with lower levels of waist circumference and fasting insulin [23, 24]. However, Yankey et al. [2] also reported that regular cannabis users had an average higher waist circumference than normal metabolic syndrome clients who did not use cannabis with significant associations between duration of cannabis use and metabolic syndrome and its elements. Each year of continued cannabis use was associated with a significant $5 \%$ increase in the odds of having metabolic syndrome and $4 \%$ increase in odds of having hypertension. Muniyappa et al. [25] opined that chronic use of cannabis was associated with visceral adiposity and adipose insulin resistance but it was not associated with insulin insensitivity, glucose intolerance and hepatic steatosis.

The most prevalent substance abused identified was alcohol. Its harmful effects can-not be overemphasized. Globally, alcohol dependence was the most prevalent of the substance use disorders, with 100.4 million estimated cases in 2016 (age-standardized prevalence 1320.8 cases per 100000 people, $95 \%$ uncertainty interval. The Disability Adjusted Life Years in Eastern sub-Saharan Africa attributed to alcohol showed the major cause as unintentional injuries and for drug use as drug use related disorders in 2016 [26]. The harmful impact of excessive alcohol consumption primarily manifests in the presence of metabolic syndrome at half the amount that the same harmful impact would manifest in individuals absent of metabolic syndrome [27]. Binge drinking is associated with mortality with adjustment for average daily consumption. The overall mortality in individuals with fatty liver disease and metabolic syndrome with excessive alcohol intake was $32.2 \%$ compared to $22.2 \%$ in individuals with non-excessive alcohol intake [27].

\section{Conclusion}

This is one of the few studies, and the first one in Kenya, seeking to understand the relationship between substance use and occurrence of metabolic syndrome. The correlation indicated herein and in literature on metabolic syndrome, its elements and substance use is a critical knowledge source in the successful design and implementation of an integrated, multidisciplinary approaches in the management of 
metabolic syndrome and substance dependency such as physical activity measures, dietary modifications, behavior change communication and psychotherapeutic approaches [28].

\section{Abbreviations}

BMI- Body Mass Index

HDL- High Density Lipoprotein

\section{Declarations}

\section{Ethics approval and consent to participate}

Ethical approval was sought from Mount Kenya University Institutional Research Ethics Committee. The research permit was received from the National Commission for Science, Technology and Innovation (NACOSTI). The researchers explained and sought informed consent from study participants with assurance of privacy and confidentiality.

\section{Consent for Publication}

Not applicable

\section{Availability of data Materials}

The datasets used and/or analyzed during the current study are available from the corresponding author on reasonable request.

\section{Competing interests}

The authors declare that they have no competing interests.

\section{Funding}

This work was made possible through a financial grant from the National Research Fund, Kenya.

\section{Authors' Contributions}

All authors were instrumental in conception, research design and data collection. SM conceptualized the idea, CM, PK, EM, FM, MN, JG, BM was instrumental in manuscript review and revision. All authors 
approved the final manuscript for submission and publication.

\section{Acknowledgements}

We would like to show our gratitude to the patients, families and staff in the participating rehabilitative centers for their cooperation and support.

\section{References}

1. Isomaa, B. O., Almgren, P., Tuomi, T., Forsén, B., Lahti, K., Nissen, M., \& Groop, L. (2001). Cardiovascular morbidity and mortality associated with the metabolic syndrome. Diabetes care, 24(4), 683-689.

2. Yankey, B. N., Strasser, S., \& Okosun, I. S. (2016). A cross-sectional analysis of the association between marijuana and cigarette smoking with metabolic syndrome among adults in the United States. Diabetes \& Metabolic Syndrome: Clinical Research \& Reviews, 10(2), S89-S95.

3. Alberti, K. G. M. M., Eckel, R. H., Grundy, S. M., Zimmet, P. Z., Cleeman, J. I., Donato, K. A., \& Smith Jr, S. C. (2009). Joint scientific statement. Circulation, 120, 1640-1645.

4. Yankey, B. (2017). The Connection between Marijuana, Cigarette Smoking and Metabolic Syndrome among Adults in the United States.

5. World Health Organization. (1985). Targets for health for all 2000. WHO Regional Office for Europe.

6. Johnston, L. D., Miech, R. A., O'Malley, P. M., Bachman, J. G., Schulenberg, J. E., \& Patrick, M. E. (2019). Monitoring the Future National Survey Results on Drug Use, 1975-2018: Overview, Key Findings on Adolescent Drug Use. Institute for Social Research.

7. Sharma, P., \& Balhara, Y. (2016). Opioid use and diabetes: An overview. Journal of Social Health and Diabetes, 4(1), 6-10.

8. Kuppili, P. P., \& Nebhinani, N. (2019). Role of integrated and multidisciplinary approach in combating metabolic syndrome in patients with severe mental illness. Indian journal of psychological medicine, 41(5), 466-471.

9. Neicun, J., Yang, J. C., Shih, H., Nadella, P., van Kessel, R., Negri, A., \& Roman-Urrestarazu, A. (2020). Lifetime prevalence of novel psychoactive substances use among adults in the USA: Sociodemographic, mental health and illicit drug use correlates. Evidence from a population-based survey 2007-2014. Plos one, 15(10), e0241056.

10. Mattoo, S. K., Chakraborty, K., Basu, D., Ghosh, A., Vijaya, K. K., \& Kulhara, P. (2011). Prevalence \& correlates of metabolic syndrome in alcohol \& opioid dependent inpatients. The Indian journal of medical research, 134(3), 341.

11. Mattoo, S. K., Nebhinani, N., Aggarwal, M., Basu, D., \& Kulhara, P. (2013). Metabolic syndrome among substance dependent men: A study from north India. Industrial psychiatry journal, 22(1), 60. 
12. Grover, S., Aggarwal, M., Dutt, A., Chakrabarti, S., Avasthi, A., Kulhara, P., \& Chauhan, N. (2012). Prevalence of metabolic syndrome in patients with schizophrenia in India. Psychiatry research, 200(2-3), 1035-1037.

13. Gardner-Sood, P., Lally, J., Smith, S., Atakan, Z., Ismail, K., Greenwood, K. E., \& Papanastasiou, E. (2015). Cardiovascular risk factors and metabolic syndrome in people with established psychotic illnesses: baseline data from the IMPaCT randomized controlled trial. Psychological medicine, 45(12), 2619-2629.

14. Walter, K. N., \& Petry, N. M. (2015). Patients with diabetes respond well to contingency management treatment targeting alcohol and substance use. Psychology, health \& medicine, 20(8), 916-926.

15. Balhara, Y. P. S., \& Kalra, S. (2017). Drug addiction and diabetes: South Asian action. The Journal of the Pakistan Medical Association, 67(6), 954-956.

16. Djoussé, L., Arnett, D. K., Eckfeldt, J. H., Province, M. A., Singer, M. R., \& Ellison, R. C. (2004). Alcohol consumption and metabolic syndrome: does the type of beverage matter?. Obesity research, 12(9), 1375-1385.

17. Gigleux, I., Gagnon, J., St-Pierre, A., Cantin, B., Dagenais, G. R., Meyer, F., \& Lamarche, B. (2006). Moderate alcohol consumption is more cardioprotective in men with the metabolic syndrome. The Journal of nutrition, 136(12), 3027-3032.

18. Yoon, Y. S., Oh, S. W., Baik, H. W., Park, H. S., \& Kim, W. Y. (2004). Alcohol consumption and the metabolic syndrome in Korean adults: the 1998 Korean National Health and Nutrition Examination Survey. The American journal of clinical nutrition, 80(1), 217-224.

19. Santos, A. C., Ebrahim, S., \& Barros, H. (2007). Alcohol intake, smoking, sleeping hours, physical activity and the metabolic syndrome. Preventive medicine, 44(4), 328-334.

20. Teixeira, P. J. R., \& Rocha, F. L. (2007). The prevalence of metabolic syndrome among psychiatric inpatients in Brazil. Brazilian Journal of Psychiatry, 29(4), 330-336.

21. Ojo, O., Wang, X. H., Ojo, O. O., \& Ibe, J. (2018). The effects of substance abuse on blood glucose parameters in patients with diabetes: A systematic review and meta-analysis. International Journal of Environmental Research and Public Health, 15(12), 2691.

22. Shah, K., Joshi, V. V., Arinze, N. C., Bodicherla, K. P., Ghimire, S., Singh, R., \& Sreeram, V. (2020). Association of Opioid Use With Cardiometabolic Disease Risk Factors: Evidence From the 2009-2018 National Health and Nutrition Examination Survey. Cureus, 12(6).

23. Penner, E. A., Buettner, H., \& Mittleman, M. A. (2013). The impact of marijuana use on glucose, insulin, and insulin resistance among US adults. The American journal of medicine, 126(7), 583-589.

24. Thompson, C. A., \& Hay, J. W. (2015). Estimating the association between metabolic risk factors and marijuana use in US adults using data from the continuous National Health and Nutrition Examination Survey. Annals of epidemiology, 25(7), 486-491.

25. Muniyappa, R., Sable, S., Ouwerkerk, R., Mari, A., Gharib, A. M., Walter, M., \& Kunos, G. (2013). Metabolic effects of chronic cannabis smoking. Diabetes care, 36(8), 2415-2422. 
26. Degenhardt, L., Charlson, F., Ferrari, A., Santomauro, D., Erskine, H., Mantilla-Herrara, A., \& Rehm, J. (2018). The global burden of disease attributable to alcohol and drug use in 195 countries and territories, 1990-2016: a systematic analysis for the Global Burden of Disease Study 2016. The Lancet Psychiatry, 5(12), 987-1012.

27. Younossi, Z. M., Stepanova, M., Ong, J., Yilmaz, Y., Duseja, A., Eguchi, Y., \& Bugianesi, E. (2019). Effects of alcohol consumption and metabolic syndrome on mortality in patients with nonalcoholic and alcohol-related fatty liver disease. Clinical Gastroenterology and Hepatology, 17(8), 1625-1633.

28. Kuppili, P. P., \& Nebhinani, N. (2019). Role of integrated and multidisciplinary approach in combating metabolic syndrome in patients with severe mental illness. Indian journal of psychological medicine, 41(5), 466-471. 